\title{
O uso da CPAP na sala de parto: por que acreditar nessa conduta?
}

A utilização da Pressão Positiva Contínua nas Vias Aéreas (CPAP) na sala de parto é de grande importância, pois atua diretamente na dizimação da ocorrência da Displasia Broncopulmonar (DBP). A DBP corresponde a uma patologia que é mais frequente em recém-nascidos (RNs) que apresentam baixo ou baixíssimo peso e que desenvolvem a Síndrome do Desconforto Respiratório. Devido a isso, precisam ser submetidos à ventilação mecânica invasiva (VMI). Os principais fatores patogênicos que se tem como base para a identificação são: prematuridade, oxigenoterapia, ventilação mecânica, edema pulmonar e infeç̧ão. A eficiência da CPAP vem sendo cada vez mais discutida e reconhecida no meio das instituições. Seu uso precoce tem sido cada vez mais divulgado pelos benefícios trazidos nesse âmbito, já que apresenta mais segurança e menos agressividade para o RN. O uso da pressão positiva contínua nas vias aéreas deve ser considerada, quando necessário, já na recepção do recém-nascido, imediatamente após o nascimento e após os primeiros cuidados. É imprescindível que todos os profissionais envolvidos tenham domínio teórico e prático para que o uso da CPAP seja cada vez mais utilizada precocemente. Este presente artigo é uma revisão de literatura cujo objetivo foi identificar os benefícios do uso da CPAP na sala de parto e em RNs prematuros.

Palavras-chave: Parto Normal; Parto Humanizado; Cesárea; Parteira.

\section{The use of CPAP in the delivery room: why believe in this behavior?}

The use of Continuous Positive Airway Pressure (CPAP) in the delivery room is of great importance, as it acts directly on the decimation of the occurrence of Bronchopulmonary Dysplasia (BPD). BPD corresponds to a pathology that is more frequent in newborns (RNs) who present low or very low weight and who develop the Syndrome of Respiratory Discomfort. Because of this, they need to undergo invasive mechanical ventilation (IMV). The main pathogenic factors that are based on the identification are: prematurity, oxygen therapy, mechanical ventilation, pulmonary edema and infection. The efficiency of CPAP has been increasingly discussed and recognized among institutions. Its early use has been increasingly disseminated by the benefits brought in this scope, since it presents more security and less aggressiveness for the RN. The use of continuous positive airway pressure should be considered, when necessary, already in the reception of the newborn, immediately after birth and after the first care. It is imperative that all professionals involved have a theoretical and practical domain so that the use of CPAP is increasingly used early. This article is a literature review aimed at identifying the benefits of using CPAP in the delivery room and in premature newborns.

Keywords: Normal birth; Humanized birth; Cesarean section; Midwife.

\section{Topic: Fisioterapia}

Reviewed anonymously in the process of blind peer.
Received: 09/08/2019

Approved: 19/08/2019

Verlândia Sales de Sousa

Faculdade Guaraí, Brasil

http://lattes.cnpq.br/0250516814003913

verlandiasousa@gmail.com

Andreia Cristina Medeiros

Faculdade Guaraí, Brasil

http://lattes.cnpq.br/2830504714922308

andreiamedeirosdh@outlook.com

Leandro Augusto Almeida

Universidade Brasil, Brasil

http://lattes.cnpq.br/0591590391105455

leandroaugustoalmeida74@gmail.com

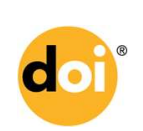

DOI: 10.6008/CBPC2236-9600.2018.002.0006

\section{Referencing this:}

SOUSA, V. S.; MEDEIROS, A. C.; ALMEIDA, L. A.. O uso da CPAP na sala de parto: por que acreditar nessa conduta?. Scire Salutis, v.8, n.2, p.54-61, 2018. DOI: http://doi.org/10.6008/CBPC2236$\underline{9600.2018 .002 .0006}$ 


\section{INTRODUÇÃO}

Neste artigo, trataremos acerca da utilização da pressão positiva contínua das vias aéreas (CPAP) na sala de parto, visto que diversos artigos, estudos e autores nos apresentam de maneira científica e coesa que o seu uso é de tamanha importância, pois atua diretamente na dizimação da ocorrência de displasia broncopulmonar (DBP) e que também nos mostrará que o uso da ventilação mecânica invasiva (VMI) e do oxigênio está associado à essa patologia. A eficiência da CPAP na sala de parto vem sendo cada vez mais debatida, discutida e reconhecida no meio das instituições e seu uso tem sido divulgado pelos benefícios trazidos nesse âmbito, pois apresenta mais segurança e menos agressividade para o recém-nascido (RN).

Segundo o DATASUS, o período neonatal compreende as quatro primeiras semanas de vida do recém-nascido (RN), ou seja, considerado desde o nascimento até os 28 dias completos. Denomina-se período neonatal o período precoce à primeira semana completa ou os sete primeiros dias de vida, e período neonatal tardio, as três semanas seguintes.

Em uma Unidade de Terapia Intensiva Neonatal (UTIN), considera-se que os RNs integram um grupo de maior risco para o desenvolvimento de diversos distúrbios pulmonares decorrentes das particularidades anatômicas, funcionais e imunológicas do sistema respiratório no período neonatal. Nas últimas duas décadas, o aumento da sobrevida destes indivíduos ocorreu devido ao avanço tecnológico e científico na área da neonatologia, que permitiu conceder melhor auxílio à saúde desses RNs.

A maioria dos recém-nascidos pré-termos (RNPT) precisa de ajuda para iniciar a transição cardiorrespiratória, necessária para a adequada adaptação à vida extrauterina. Dados da Rede Brasileira de Pesquisas Neonatais, composta por 20 centros universitários públicos, indicam que, nos anos de 2012-2014, dos 4.352 nascidos vivos de muito baixo peso, com idade gestacional entre 23-33 semanas, sem malformações, $62 \%$ foram ventilados com máscara facial ou cânula traqueal e $6 \%$ receberam ventilação acompanhada de massagem cardíaca e/ou medicações na sala de parto.

Estudo da NICHD Neonatal Research Network, com dados referentes a 9.565 neonatos com idade gestacional entre $22-28$ semanas e peso de $401-1.500 \mathrm{~g}$, nascidos entre $2003-2007$, mostra que $67 \%$ deles receberam ventilação com pressão positiva (VPP), $8 \%$ necessitaram de massagem cardíaca e $5 \%$ de medicações na sala de parto (GUINSBURG et al., 2016).

Observa-se, portanto, que a necessidade de VPP e a de manobras avançadas de reanimação na sala de parto são bastante frequentes em RNPT, especialmente naqueles de muito baixo peso. A elevada necessidade de ajuda para iniciar a respiração efetiva em sala de parto, ou seja, efetuar a transição para o ambiente extrauterino, e de reanimação propriamente dita nos RNPT se deve, de modo geral, à sua imaturidade global do ponto de vista anatômico e fisiológico (GUINSBURG et al., 2016).

Com a confirmação de que a retinopatia da prematuridade estava relacionada à hiperóxia, o uso de oxigênio tornou-se restrito nas unidades neonatais, provocando diminuição na incidência da doença, embora aumento da mortalidade. Em 1971, Gregory e seus colaboradores introduziram o método de pressão positiva contínua das vias aéreas - CPAP - na respiração assistida em recém-nascidos. Tal fato levou a uma queda dramática na mortalidade, concomitantemente a um aumento na incidência da doença, já que o método 
promoveu um incremento efetivo na tensão arterial de oxigênio, mesmo quando fornecido em baixas concentrações.

A CPAP tem se mostrado, nas últimas décadas, um aliado no tratamento de questões respiratórias e principalmente no que comtempla o âmbito neonatal, visto que através da pressão transpulmonar positiva é possível aumentar a capacidade residual funcional (CRF). Todavia, devemos nos atentar aos custos que a DBP gera ao Sistema Único de Saúde (SUS), devido ao período de internação do RN, causado pelas lesões pulmonares, assim sendo o uso da CPAP visa reduzir a incidência de DPB e consequentemente diminuirá os gastos hospitalares. Entretanto, é imprescindível que a equipe esteja devidamente preparada para acompanhar toda a técnica, além de possuir total domínio científico da aplicação, do procedimento e manejo técnico.

Dentro da equipe multidisciplinar, o fisioterapeuta tem se tornado cada vez mais indispensável nos processos de assistência e suporte ventilatório aos RNs, sendo nos dias atuais, considerado o principal membro da equipe na instalação e monitorização da VNI em muitas instituições. Logo, é uma assistência que contribui para prevenir, reverter ou diminuir disfunções respiratórias, proporcionando uma maior funcionalidade e o bem estar ao RN.

Vale salientar que a utilização da CPAP no tratamento do RN vem ganhando mais espaço e maior preferência, principalmente por contribuir contra o aparecimento de DBP por se tratar de um tratamento menos agressivo que a VMI, visto que as lesões pulmonares são geradas em consequência da VMI devido às pressões inspiratórias e o oxigênio por causa dos radicais livres.

A CPAP é a única tecnologia que foi direcionada ao âmbito neonatal que de maneira comprovada tem contribuído diretamente para a melhora do prognóstico respiratório reduzindo o tempo de entubação traqueal e barotrauma. O uso da CPAP nasal no lugar da VMI tem se mostrado diretamente ligado à redução da incidência de DBP.

A utilização da CPAP na sala de parto tem sido realizada com o objetivo de diminuir o uso da VMI e reduzir a probabilidade do aparecimento e desenvolvimento da DBP. De acordo com os estudos que estão disponíveis, é relatado que o uso da VMI tem se mostrado como um dos principais causadores de lesões pulmonares que é apresentada em prematuros, pois o uso da VMI é o principal indicador de causa de barotrauma, e essa lesão tem causado enormes repercussões dentro das equipes que fazem uso da VMI em neonatologia.

O uso da CPAP nasal vem adquirindo cada vez mais espaço e prestígio, visto que através da pressão transpulmonar positiva acontece à elevação da capacidade residual funcional (CRF), que acaba se tornando uma forma de depósito de oxigênio que será utilizada em situações onde o fluxo inspiratório for pequeno ou inexistente. A CPAP nasal é uma terapêutica de excelência e que possui resultados satisfatórios, e merece total atenção e mérito. O objetivo desse estudo é realizar uma revisão de literatura para identificar os benefícios da CPAP em sala de parto e em recém-nascidos prematuros. 


\section{METODOLOGIA}

A metodologia utilizada na construção desta revisão de literatura baseia-se no conteúdo encontrado em 13 artigos científicos que foram selecionados através das plataformas PubMed, Scielo e JPED onde todos os artigos encontrados e escolhidos abordam a utilização da CPAP em RN e em casos de prematuridade. Os autores trazem métodos e técnicas que são utilizados em pacientes prematuros e que necessitam de suporte no âmbito do sistema respiratório.

\section{DISCUSSÃO TEÓRICA}

A CPAP estimula a pressão positiva contínua nas vias aéreas, de poucos centímetros da água ( $\mathrm{cmH2O})$, mantenho os valores predeterminados durante todo o ciclo respiratório e a respiração realizada inteiramente pelo paciente. É notável e bastante considerável que a aplicação da CPAP nasal precoce reduz a necessidade de utilizar a VMI, sendo utilizado para tornar a extubação mais fácil. Gomes et al. (2015) afirma que:

Nesta modalidade, o princípio da ventilação fisiológica realizado com pressão negativa é modificado para pressão positiva, onde o RN é obrigado a respirar o ar que está contido no circuito, sendo esse, normalmente enriquecido com oxigênio. Durante a fase expiratória, o ar liberado é expelido por uma válvula expiratória a qual possui um sistema graduado de PEEP, que tem por objetivo aumentar o período de troca de oxigênio entre os alvéolos e os capilares.

A ventilação mecânica não invasiva (VMNI) é uma terapêutica alternativa que promove uma ventilação mecânica sem a utilização de uma aérea artificial (intubação endotraqueal ou traqueostomia). Em casos de insuficiência respiratória, em que somente a oxigenoterapia não é suficiente para reverter quadros de hipoxemia e tem-se desconforto respiratório associado (tiragens, batimento de asa de nariz, balanceio de cabeça, gemência, cianose e taquicardia), presença de apneia recorrente com quedas de saturação e bradicardia, presença de áreas de atelectasia que prejudiquem a oxigenação e radiografia de tórax indicando síndrome do desconforto respiratório (SDR), a utilização de VMNI constitui uma opção antes que se proceda a intubação orotraqueal (IOT) e VMI, permitindo a respiração espontânea.

No processo de VMNI, têm-se como objetivos primordiais, um processo respiratório livre de esforços demasiados e a diminuição da necessidade de recorrer à VMI além de almejar também melhores trocas gasosas e redução do tempo de internação hospitalar, além de diminuir o risco de DBP, barotrauma e pneumonia.

É de suma importância ressaltar que a VMNI tem como objetivo diminuir e evitar as taxas de morbidade e mortalidade em RN, e já há alguns anos foi possível perceber que o interesse em aplicação de VMNI vem crescendo e ganhando cada vez mais espaço, tendo em vista que por meio da VMNI há maior proteção e conservação da fala, deglutição, tosse, umidificação das vias aéreas e possibilidade e facilidade de alimentação. Vale a pena lembrar que a VMNI ganhou espaço por ser vista como uma alternativa terapêutica e por ser um mecanismo bastante seguro e eficiente, que apresenta baixos custos, reduz o tempo de internação e possui a menor taxa de mortalidade (GOMES et al., 2015). 
A CPAP tem ganhado grande espaço e vem sida bastante recorrida quando se trata de doenças respiratórias em âmbito neonatal, pois por meio da pressão transpulmonar positiva é possível elevar a CRF, que tem a função de servir como uma espécie de depósito de oxigênio que será utilizado nos períodos em que não houver fluxo inspiratório. Entretanto, devido aos índices de prevalência de DBP em prematuros, as UTIs neonatais correspondem a maior parcela de gastos hospitalares.

Ao analisarmos os materiais e estudos disponíveis para pesquisa, nos deparamos com autores e pesquisadores que incentivam e indicam o uso da CPAP na sala de parto sendo utilizado como uma espécie de estratégia para diminuir o suporte da VMI e consequentemente de acordo com o que se tem pesquisado diminuir a probabilidade do desenvolvimento da DBP.

O pulmão do recém-nascido prematuro é geralmente deficiente em surfactante, o que desencadeia a atelectasia alveolar e a diminuição da complacência pulmonar. O uso de VMI para o recrutamento dos alvéolos atelectáticos pode causar lesão pulmonar. A melhor maneira de prevenir a lesão pulmonar é evitar o parto prematuro. Uma vez que o parto não possa ser evitado, deve-se usar o corticosteroide pré-natal para acelerar o amadurecimento alveolar e capilar no pulmão desses recém-nascidos.

A aplicação de CPAP em RNPT ajuda a manter os alvéolos dos pulmões imaturos e deficientes em surfactante não colapsados, evitando o atelectrauma. Metanálise de três ensaios clínicos e um estudo observacional, que analisaram o uso de CPAP versus intubação e ventilação na sala de parto em 2.782 neonatos $<32$ semanas, mostrou que CPAP reduz a necessidade de ventilação mecânica e de surfactante exógeno no período neonatal, sem elevar a incidência de pneumotórax. CPAP iniciado em sala de parto diminui a dependência de oxigênio com 36 semanas ou óbito hospitalar: para cada 25 RNPT que recebem CPAP, em vez de serem intubados e ventilados na sala de parto, uns bebês a mais pode sobreviver sem displasia broncopulmonar com 36 semanas de idade gestacional corrigida. Segundo Suguihara (2005),

Além de recrutar alvéolos e aumentar o volume pulmonar, a CPAP reduz as distorções torácicas e estabiliza a caixa torácica, como também diminui a incidência de apneia obstrutiva e aumenta a excreção de surfactante. $O$ uso da CPAP, por ser um procedimento menos invasivo do que a ventilação mecânica, vem sendo estudado como tratamento precoce, muitas vezes ainda na sala de parto, em prematuros extremos.

Na reanimação do RNPT em sala de parto, as pesquisas ainda não responderam à questão relativa à concentração de oxigênio ideal durante a ventilação. A hipóxia se associa a lesões disfuncionais em todos os sistemas biológicos, que acabam por resultar em falência de múltiplos órgãos e morte. A hiperóxia, por sua vez, gera radicais livres, que desencadeiam oxidação enzimática, inibição de síntese proteica, inibição da síntese de DNA e peroxidação lipídica, com lesão tecidual difusa mais acentuada nos RNPT, pois seus mecanismos de proteção antioxidantes são imaturos. Assim, por um lado, o uso de ar ambiente pode não ser suficiente para que tais pacientes atinjam uma oxigenação adequada; por outro lado, o emprego de oxigênio a $100 \%$ é deletério, contribuindo para as lesões inflamatórias em nível sistêmico.

Com base no conhecimento existente até o momento, recomenda-se não iniciar a reanimação com concentrações elevadas de oxigênio. O uso de concentrações de oxigênio $\geq 65 \%$ em RNPT, comparado a concentrações entre $21-30 \%$, não leva à melhora na sobrevida, displasia broncopulmonar, hemorragia peri- 
intraventricular e retinopatia da prematuridade 51-56, havendo um aumento de marcadores de estresse oxidativo (GUINSBURG et al., 2016).

Até o presente momento diversas instituições vinculadas à saúde e a pesquisa têm demonstrado que a aplicação da CPAP nasal é factível e segura quando essa aplicação ocorre logo após o nascimento em prematuros com idade gestacional inferior a 28-30 semanas. De fato a indicação da CPAP nasal vem ganhando cada vez mais força e espaço dentro das instituições, visto que os profissionais presentes na equipe que fazem o uso dessa terapêutica contemplam e demonstram maior satisfação com os resultados obtidos através do uso da CPAP nasal do que através do uso da VMI, entretanto vale salientar que existem casos de diversos graus de complexidade e particularidade onde cada terapêutica pode surtir efeito de acordo com o quadro clínico do paciente.

Para Procianoy (1998), “a DBP é uma doença de origem multifatorial, que ocorre quase exclusivamente em recém-nascidos prematuros que tenham sido ventilados mecanicamente". A DBP corresponde a uma patologia que é mais frequente em RNs que apresentam baixo ou baixíssimo peso e que desenvolvem a síndrome do desconforto respiratório, e devido a isso precisam ser submetidos à VMI. Os principais fatores patogênicos que se tem como base para a identificação são: prematuridade, oxigenoterapia, ventilação mecânica, edema pulmonar e infecção (PROCIANOY, 1998).

A incidência da DBP é inversamente proporcional à idade gestacional e ao peso de nascimento. Sua ocorrência é pouco comum em neonatos com idade gestacional superior a 34 semanas, apesar de existirem casos descritos em recém-nascidos a termo. Com os avanços na assistência perinatal, a incidência da forma grave ou clássica da DBP diminuiu consideravelmente. Além disso, os relatos na literatura são extremamente variáveis de centro para centro, devido às diferenças da população estudada, à falta de padronização das estratégias ventilatórias empregadas no tratamento da doença pulmonar aguda, à grande variabilidade no manuseio da oferta hídrica e na prática de cuidados de RN criticamente doentes e aos diferentes critérios diagnósticos utilizados para definir a doença (MONTE et al., 2005).

A patogênese da DBP é multifatorial. Acredita-se que esses diversos fatores atuem de forma aditiva ou sinérgica, gerando inflamação e lesão pulmonar. A agressão ao tecido pulmonar em desenvolvimento resulta em fibrose e desorganização do processo maturativo normal. Uma série de eventos perinatais tem sido associada à ocorrência da DBP, com especial atenção ao processo de desenvolvimento do sistema respiratório. O desenvolvimento pulmonar completa-se nos primeiros anos de vida, de tal modo que mesmo indivíduos nascidos a termo estão vivenciando intensamente o período de alveolarização do tecido pulmonar.

Os nascimentos antes do termo podem ocorrer durante a fase canalicular do desenvolvimento pulmonar, que compreende o período da 17으 a 24 semana de gestação, ou durante as fases de saculação terminal e alveolar (a partir da 25오 semana). Na fase canalicular, ocorre a maturação das vias condutoras pulmonares e inicia-se o desenvolvimento das unidades respiratórias terminais, que irão amadurecer nas fases sacular e alveolar, preparando o pulmão para a troca gasosa. A lesão durante a formação do parênquima pulmonar pode resultar em uma alteração na progressão do processo de alveolarização, 
resultando num quadro de simplificação alveolar. Dessa forma, a DBP representa a ruptura do processo normal de desenvolvimento e reparação dos pulmões em resposta à lesão aguda pulmonar (MONTE et al., 2005).

A DBP apareceu pela primeira vez na literatura em 1967, através de Northway e colaboradores, onde ele afirmava que RNs prematuros que eram portadores da síndrome do desconforto respiratório foram submetidos à ventilação com pressão positiva de maneira intermitente e receberam grandes e altas quantidades de oxigênio inspirado (PROCIANOY, 1998). E ainda segundo Procianoy (1998):

Inicialmente, foi descrita em quatro estágios clínico-radiológicos distintos. Os estágios I e II eram indistinguíveis da doença de membrana hialina e ocorriam nos primeiros dez dias de vida. Os estágios III e IV caracterizavam a sua cronicidade, sendo, o quarto estágio, o mais severo, podendo persistir por mais de 28 dias.

Vinte anos após 1967, Northway e seus colaboradores informaram que sintomas respiratórios consideráveis e anormalidades funcionais continuaram aparecendo de forma significativa em adolescentes e jovens adultos num grupo de pacientes que sobreviveram a DBP nos primeiros dias de nascimento, e isso nos indica que a lesão pulmonar quando obtida de forma precoce pode ter consequências em longo prazo (SADECK, 2007). Procianoy (1998) relata que:

Os recém-nascidos com DBP apresentam um quadro de insuficiência respiratória que persiste além da duração normal da doença base. Em geral, é um recém-nascido prematuro de muito baixo peso que desenvolve doença de membrana hialina, necessitando de ventilação mecânica, e que persiste sendo ventilado por um período muito prolongado. Pode-se suspeitar que um paciente está desenvolvendo DBP quando a necessidade de ventilação mecânica ou a dependência de oxigênio persiste por mais de 10 a 14 dias.

Fica claro e evidente que o uso da VMI tem se apresentado como um dos principais fatores de lesão pulmonar presente em prematuros, pois o uso da VMI é o principal indicador de causa de barotrauma, e tal lesão tem causado enormes repercussões dentro das equipes que fazem uso da VMI em neonatologia (GONZAGA et al., 2007).

Ao direcionarmos o nosso olhar para RNs, a Síndrome do Desconforto Respiratório apresenta ativação significativa de fagócitos nas três primeiras horas de início da VMI, sendo utilizado surfactante ou não, e isso demonstra que o uso da VMI pode ser um fator extremamente considerável dessa resposta inflamatória sistêmica.

O RN, e principalmente o prematuro, além de estar exposto à maior produção de radicais tóxicos de oxigênio, não apresenta em seus sistemas de defesa antioxidantes totalmente desenvolvidos. O uso da CPAP na sala de parto tem sido para tentar diminuir o uso da VMI e diminuir a probabilidade do aparecimento e desenvolvimento da DBP. Assim, ao invés da VMI, tem sido estreitamente associado a redução da taxa de DBP. Tem-se demonstrado a fácil aplicação da CPAP nasal precoce. Seu uso reduz a necessidade de VMI e é frequentemente usado para facilitar a extubação e tratar a apneia da prematuridade. Uma técnica proveniente e pertencente a Dr. Wung, que consistia em um aparato nasal que possuía capacidade de imobilização, e por meio dessa peça e do uso da CPAP, Wung conseguia diminuir a exposição dos RNs à VMI o que resultava em menor índice e probabilidade de DBP. 
É clara a forma como o sucesso da aplicação da CPAP nasal vem ganhando cada vez mais espaço, pois além de ser uma técnica mais simples para manuseio e aplicação, demanda menos gastos e trata da insuficiência respiratória em RNs. É a única tecnologia recentemente introduzida no cuidado neonatal que comprovadamente melhora o prognóstico respiratório na medida em que diminui o tempo de entubação traqueal e a agressão broncopulmonar.

Ao se tratar da CPAP, além de aplicar uma pressão continua para prevenir o colapso dos alvéolos e aumentar o volume pulmonar, também é função da CPAP diminuir as distorções torácicas. A CPAP mostrase como um procedimento menos invasivo e menos agressivo que a VMI, e por causa disto vem ganhando maior destaque e no estudo para o tratamento precoce proveniente desde a sala de parto em casos de prematuros extremos.

\section{CONCLUSÃO}

Com base no que foi supramencionado nos parágrafos anteriores, é possível constatar que de acordo com cada um dos artigos citados e dos autores mencionados, o uso da CPAP nasal na sala de parto tem sido extremamente eficaz e tem atingido resultados relevantes. Os dados e as informações trazidas pelos autores reforçam que o uso da CPAP vem ganhando cada vez mais espaço e notoriedade, pois tem se mostrado e se confirmado cada vez mais seguro e eficiente para atender as demandas que são recebidas. Desse modo, o uso da CPAP deve ser levado em consideração e deve ser considerado de acordo com a situação determinada, porém é clara a forma como essa terapêutica se apresenta confiável e segura.

\section{REFERÊNCIAS}

ALMEIDA, M. F. B.; GUINSBURG, R.. A reanimação do prematuro extremo em sala de parto: controvérsias. Jornal de Pediatria, Rio de Janeiro, p.3-15, 2005.

CARVALHO, C. G.; SILVEIRA, R. C.; PROCIANOY, R. S.. Lesão pulmonar induzida pela ventilação em recém-nascidos prematuros. Revista Brasileira de Terapia Intensiva, São Paulo, v.25, n.4, p.319-326, 2013.

GOMES, C. T. F.; MALVÃO, M. M.. Ventilação não invasiva em UTI neonatal: revisão bibliográfica. Monografia (Graduação em Fisioterapia) - Faculdade de Pindamonhangaba, Pindamonhangaba, 2015.

GONZAGA, A. D.; FIGUEIRA, B. B. D.; SOUSA, J. M. A.; CARVALHO, W. B.. Tempo de ventilação mecânica e desenvolvimento de displasia broncopulmonar. Revista da Associação Medicina Brasileira, São Paulo, v.1, n.53, p.6467, 2007.

GUINSBURG, R.; ALMEIDA, M. F. B.. Reanimação do prematuro <34 semanas em sala de parto: diretrizes 2016 da Sociedade Brasileira de Pediatria. São Paulo: SBP, 2016.
LOPES, J. M. A.. O uso do CPAP na assistência ventilatória neonatal. Jornal de Pediatria, p.329-330, 2000.

MONTE, L. F. V.; SILVA FILHO, L. V. F.; MIYOSHI, M. H.; ROZOV, T.. Displasia broncopulmonar. Jornal de Pediatria, Rio de Janeiro, v.81, n.2, 2005.

PROCIANOY, R. S.. Displasia broncopulmonar. Jornal de Pediatria, Porto Alegre, p.95-98, 1998.

RODRIGUES, F. P. M.. Importância dos radicais livres de oxigênio no período neonatal. Jornal da Pediatria, Rio de Janeiro, v.74, n.2, p.91-98, 1998.

SADECK, L. S. R.. A nova displasia broncopulmonar: diagnóstico. In: CONGRESSO BRASILEIRO DE PERINATOLOGIA, 19. Anais. São Paulo, 2007.

SUGUIHARA, C.; LESSA, A. C.. Como minimizar a lesão pulmonar no prematuro extremo: propostas. Jornal de Pediatria, Rio de Janeiro, v.81, p.69-78, 2005.

A CBPC - Companhia Brasileira de Produção Científica (CNPJ: 11.221.422/0001-03) detém os direitos materiais desta publicação. Os direitos referem-se à publicação do trabalho em qualquer parte do mundo, incluindo os direitos às renovações, expansões e disseminações da contribuição, bem como outros direitos subsidiários. Todos os trabalhos publicados eletronicamente poderão posteriormente ser publicados em coletâneas impressas sob coordenação da Sustenere Publishing, da Companhia Brasileira de Produção Científica e seus parceiros autorizados. Os (as) autores (as) preservam os direitos autorais, mas não têm permissão para a publicação da contribuição em outro meio, impresso ou digital, em português ou em tradução. 